Document downloaded from:

http://hdl.handle.net/10251/37520

This paper must be cited as:

Peris Tortajada, M.; Escuder Gilabert, L. (2013). On-line monitoring of food fermentation processes using electronic noses and electronic tongues: A review. Elsevier Masson. doi:10.1016/j.aca.2013.09.048.

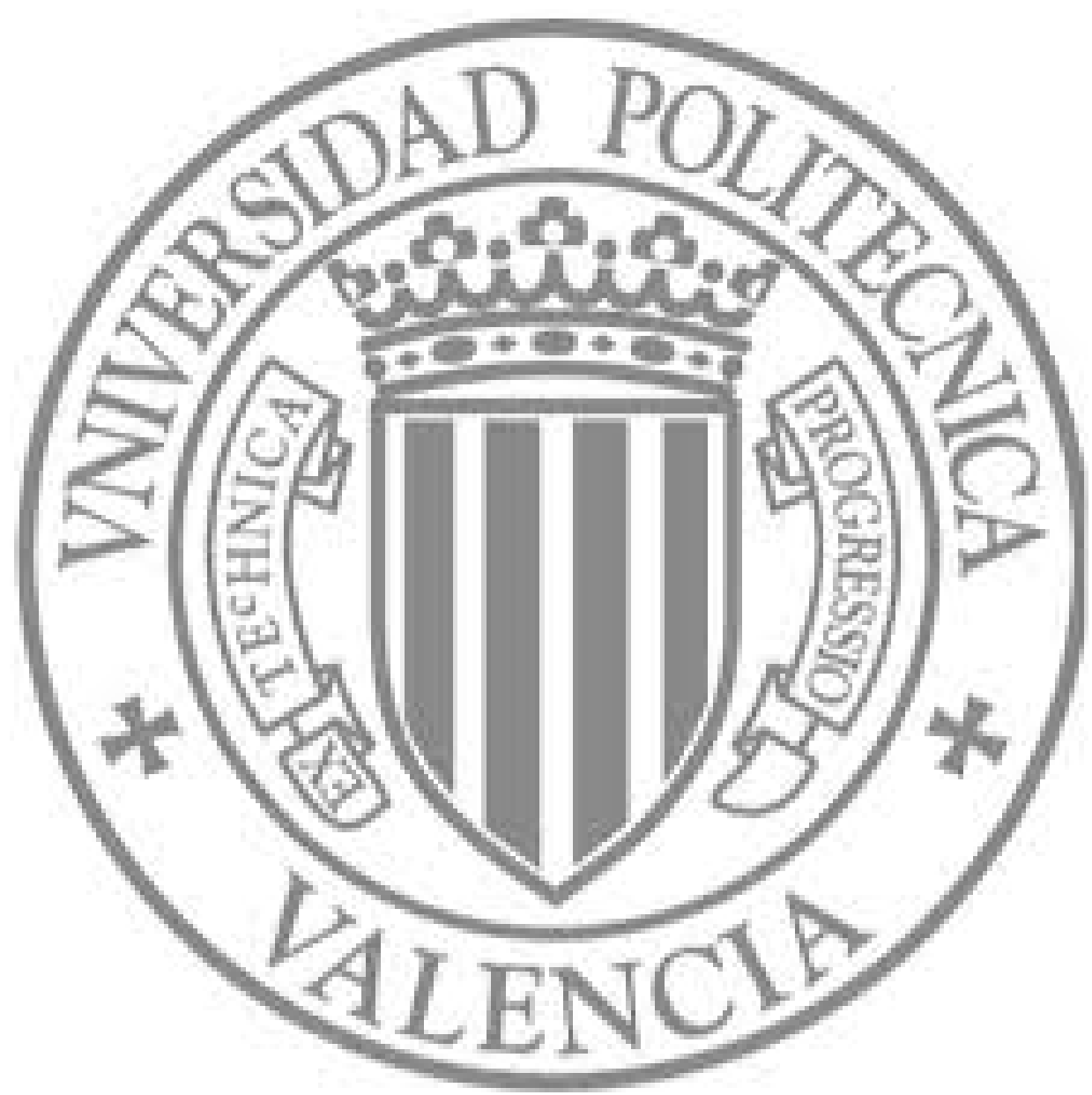

The final publication is available at

http://dx.doi.org/10.1016/j.aca.2013.09.048

Copyright Elsevier Masson 


\title{
On-line monitoring of food fermentation processes using electronic noses and electronic tongues: A review
}

\author{
Miguel Peris ${ }^{a *}$, Laura Escuder-Gilabert ${ }^{b}$ \\ a Departamento de Química, Universidad Politécnica de Valencia, 46071 Valencia, \\ Spain \\ b Departamento de Química Analítica, Universitat de Valencia, C/ Vicente Andrés \\ Estellés s/n, E-46100 Burjasot, Valencia, Spain
}

\section{ABSTRACT}

Fermentation processes are often sensitive to even slight changes of conditions that may result in unacceptable end-product quality. Thus, close follow-up of this type of processes is critical for detecting unfavorable deviations as early as possible in order to save downtime, materials and resources. Nevertheless the use of traditional analytical techniques is often hindered by the need for expensive instrumentation and experienced operators and complex sample preparation. In this sense, one of the most promising ways of developing rapid and relatively inexpensive methods for quality control in fermentation processes is the use of chemical multisensor systems. In this work we present an overview of the most important contributions dealing with the monitoring of fermentation processes using electronic noses and electronic tongues. After a brief description of the fundamentals of both types of devices, the different approaches are critically commented, their strengths and weaknesses being highlighted. Finally, future trends in this field are also mentioned in the last section of the article.

Keywords:

Electronic nose

Electronic tongue

Fermentation monitoring

Food analysis

Biotechnological process

* Corresponding author.

Tel.: +34 963877007; Fax: +34 963877349

e-mail address: mperist@qim.upv.es (M. Peris) 


\section{Introduction}

The monitoring of many production processes in which some chemical parameters vary in the course of time is key to comply with quality control regulations. Typical examples are fermentation processes producing food or beverages. These processes usually take a long time (even over 20 days in some cases), so that their continuous monitoring is critical to avoid deviations caused by microbiological contamination or system malfunctions. Furthermore, fermentations are usually sensitive to certain changes and require accurate and continuous control to ensure the feasibility of the processes.

However, the control of these processes is still suboptimal. Only a few parameters $\left(\mathrm{pH}, \mathrm{O}_{2}, \mathrm{~T}, \ldots\right)$ can be easily monitored in situ. Additional information required for process control and decision making is usually based on data produced by infrequent sampling, which in addition often has a significant time delay from sampling to obtaining the final result. Generally, analyses of target compounds are performed off-line by wet chemical assays often involving enzymatic reactions or separation techniques, such as high-performance liquid chromatography (HPLC) or gas chromatography (GC). Unfortunately, these assays require a tedious sample preparation that is usually time consuming.

The ideal method for fermentation process control should enable direct rapid, precise, and accurate determination of several target compounds, with minimal or no sample preparation and reagent consumption. Chemical sensors might then be a suitable alternative, mainly due to their well-known characteristics, such as relatively simple instrumentation, low prices, minimal sample preparation and easy automation of measurements. All these features would make chemical sensors an interesting tool for process control were it not for the fact that their use in fermentation media is often hindered by their insufficient selectivity. For example, only $\mathrm{pH}$ and oxygen probes are routinely used in bio-reactors. In fact, the literature shows many references dealing with the lack of suitable sensors for on-line fermentation monitoring.

In this sense, one of the most promising approaches allowing to overcome the aforementioned selectivity problems is the utilization of chemical multisensor systems (instead of discrete sensors), namely electronic noses and electronic tongues. They are capable to perform both qualitative analysis (classification or recognition of multicomponent media) and quantitative analysis (components' concentrations), thus becoming particularly suitable for the continuous monitoring of fermentation processes. In the following sections of this work, major contributions of e-nose and e-tongue applications in the monitoring of this type of processes will be commented. 


\section{Electronic noses \& tongues}

An electronic nose (e-nose) is a machine that is designed to detect and discriminate among complex odors using a sensor array. The sensor array consists of broadly tuned (non-specific) sensors made of a variety of odor-sensitive biological or chemical materials. An odor stimulus generates a characteristic fingerprint (or smellprint) from the sensor array. Patterns or fingerprints from known odors are used to construct a database and train a pattern recognition system so that unknown odors can subsequently be classified and identified.

Electronic nose instruments are composed of three elements, namely: (i) a sample handling system, (ii) a detection system, and (iii) a data processing system (Figure 1a). All of them are described in detail in the specialized literature [1, 2]. Some recent reviews have also appeared dealing with the state of the art of e-noses [3, 4].

Electronic tongues (e-tongues) can be considered as analytical instruments that artificially reproduce the taste sensation. These devices are typically array of sensors coupled to chemometric processing used to characterize complex liquid samples [5]. If properly configured and trained (calibrated), the e-tongue is capable of recognizing the qualitative and quantitative composition of multispecies solutions of different natures. The IUPAC technical report on the topic defines it as "a multisensor system, which consists of a number of low-selective sensors and uses advanced mathematical procedures for signal processing based on the pattern recognition (PARC) and/or multivariate data analysis" [6].

Similarly to an e-nose, an e-tongue is also composed of three elements (Figure 1b): (i) automatic sampler (although it is not a necessary component), (ii) array of chemical sensors with different selectivity, and, (iii) software with the appropriate algorithm to process the signal and get the results [3]. A more detailed description of such components is beyond the scope of this review and can be found in the literature [5 and references therein, 7-11].

As Vlasov et al. [6] pointed out, the general concepts of the electronic tongue and electronic nose used for analysis of liquids and gases, respectively, are similar. The rationale for application of these low-selective sensors is based on an analogy to biological organization of the olfactory and taste systems in mammals. In the regions of the nose and tongue, there are millions of nonspecific receptors that respond to different substances present in the gas and liquid phases. However, only about 100 different types of olfactory receptors are known, while several dozens were identified in 
the taste buds on tongues of mammals. The taste and odor signals from the receptors are transmitted to the brain where they are processed by nets of neurons. As a result, the image of the sensed object is created.

The strengths of both the electronic nose and tongue include high sensitivity and the fact that they are easy to build, cost-effective and provide a short time of analysis. Therefore, these devices are becoming more and more popular as objective automated non-destructive techniques to characterize flavors developed in the above mentioned processes. Nevertheless, it should be remarked that there is much research still to be done especially with regard to sensors technology, data processing, interpretation of results and validation studies. For instance, common problems associated with the sensor arrays are temporal drift, contamination of sensing elements and therefore some calibration instability. Other important missing aspects in some applications are the lack of long-term studies, limited feasibility studies with concurrent poor validation (especially in terms of predictive ability) as well as intermediate precision conditions (e.g., using different operators or different devices). Nevertheless, it must be mentioned that researchers are becoming increasingly aware of these problems and are addressing them in more or less extension in the recent literature.

\section{Fermentation monitoring}

\subsection{Using e-noses}

Several successful applications of electronic noses to the monitoring of flavor and/or aroma components along a fermentation process have been published. The main features of such applications are summarized in Table 1. Additionally, three interesting reviews on applications of e-nose technologies have recently appeared covering -among others- fermentations in general $[12,13]$ and in brewery in particular [14].

The detection system of e-noses usually consists of an array of chemical gas sensors (i.e., piezoelectric, electrochemical, optical and calorimetric sensors) or biosensors (i.e., sensors that incorporate a biological sensing element). Recently, new technologies such as mass spectrometry (MS) and ion mobility spectrometry (IMS) have entered in this field [2]. As can be observed in Table 1, electrochemical sensors, mainly metal oxide semiconductors (MOS) and metal oxide semiconductor field-effect transistors (MOSFET) but also conducting polymers (CPs) sensing materials, are the most used sensors in fermentation monitoring applications. Additionally, MS-based enoses have proved to be useful in this research area. Regarding the sample handling 
system, static head space (SHS) is undoubtedly the most popular technique to introduce the volatile compounds into the e-nose detection system. The main features of e-nose applications shown in Table 1 are described thereafter.

Aroma production along grape must fermentation has been monitored during the process [15]. In this study, the muscatel aroma was chosen because the profile formed as a result of yeast metabolism is complex, being composed of many compounds. These differ from each other in concentration, chemical and organoleptic properties and contribute to the overall muscatel aroma. This, therefore, was a challenging project. A commercially available e-nose consisting of 32 organic conducting polymer-based sensors was used. Data analysis was carried out by principal component analysis (PCA). The authors found that without sample pretreatment, the nose could only detect ethanol production, while for small quantities of muscatel a selective enrichment step was needed. Once this was done, the electronic nose was able to discriminate samples based on aroma content. However, enrichment increases the time and effort required for analysis, which could prove to be a major disadvantage to this approach. An additional problem is the fact that there is competition between the aroma compounds and ethanol for detection by the sensor. As ethanol occurs in higher concentration, it will be detected preferentially to the aroma.

A further complication is that ethanol may also interfere during the headspace sampling. Ethanol acts as a co-solvent in the aqueous wine-must matrix and so the activity coefficient of the hydrophobic aroma compounds in the aqueous-phase is lowered, resulting in a decreased partitioning into the sample headspace [15]. This can lead to erroneous results with the electronic nose.

E-noses have been also applied to bioprocess monitoring where microbiological processes are involved in food production, i.e. to screen the aroma generation of lactic acid bacteria strains in the production of cheese and other fermented dairy products. In a paper by Marilley et al. [16], PCA inspection of a mass spectrometry based e-nose data allowed the discrimination between 7 different genotypes strains of Lactobacillus casei isolated from Gruyère cheeses (very important in order to differentiate bacterial populations in cheese samples and to screen for new aroma-producing strains). This classification of strains based on the production of volatile compounds was in conformity with the classification obtained with the repetitive extragenic palindromic polymerase chain reaction (REP-PCR) molecular method. Gutiérrez-Méndez et al. [17] used a metal oxide semiconductors based e-nose to screen the aroma generation of Lactococcus lactis strains isolated from different dairy, non-dairy and industrial sources for their potential use in starter cheese cultures. PCA evaluation of e-nose data showed 
a clear separation of 4 sample groups based on their odor intensity scores (yogurt-like and Fresco cheese-like), but not based on the isolation source.

During the winemaking process, unpleasant organoleptic taints arise from Brettanomyces yeasts spoilage. The two main components of the taint are 4ethylphenol (4EP) and 4-ethylguaiacol (4EG). The existing procedures to monitor spoilage due to Brettanomyces/Dekkera sp. are time-consuming and expensive, making it difficult for winemakers to monitor their wines at all stages of production. Consequently, there is a need for a rapid and cost-effective screening method to monitor the levels of 4EP and 4EG in wine. In this way, Cynkar, et al. [18] used a MSbased e-nose together with PCA and stepwise linear discriminant analysis (SLDA). On the other hand, Berna et al. [19] compared the performance of a MOS sensor based enose and a MS-based e-nose. GC-MS was used for quantification and prediction purposes. Following ethanol removal and solid-phase microextraction (SPME) sample handling, the limits of detection of a MOS based e-nose were determined as $44 \mathrm{\mu g} \mathrm{L}^{-1}$ for $4 \mathrm{EP}$ and $91 \mathrm{\mu g} \mathrm{L}^{-1}$ for $4 \mathrm{EG}$ (values significantly lower than the reported human sensory thresholds). Partial least squares (PLS) regression of MOS based e-nose signals against known levels of 4EP and 4EG in 46 Australian red wines showed that such device was unable to identify Brettanomyces spoilage reliably because of the response of the gas sensors to inter-sample variation in VOCs other than 4EP and 4EG. Conversely, the MS-based e-nose (static headspace sample handling without ethanol removal but selecting a window scan excluding ethanol derived ions) was capable of reliably estimating concentrations of 4EP higher than $20 \mathrm{\mu g} \mathrm{L}^{-1}$ and good PLS correlations were obtained between estimates of 4EP and 4EG concentrations with the concentrations determined by conventional GC-MS.

Another interesting contribution deals with tea fermentation. During black tea manufacturing, tea leaves pass through a fermentation in which the grassy smell is transformed into a floral smell. Optimum fermentation is then extremely crucial in deciding the final quality of finished tea and it is very important to terminate the fermentation process at the right time. Bhattacharya et al. [20, 21] presented a study on real-time smell monitoring of black tea during the fermentation process using an e-nose (8 MOS sensors array) as well as prediction of the correct fermentation time. Different time-delay neural networks (TDNNs) and self-organizing map (SOM) methods for the prediction of optimum fermentation were used and both the methods appear to be suitable for the purpose. However, the combined SOM- and TDNN-based prediction algorithm proved to be the best alternative as the computational complexity is relatively less. The results (correlated with those from colorimetric tests and human expert evaluation) showed excellent promise for the instrument to be used for the on-line 
prediction of optimum fermentation time by the industry. This work has been recently updated by the same group of Indian researchers [22].

In the wine industry it is important to monitor time-related changes that occur during wine fermentation [23]. Eight micro-fermentation trials conducted in the Valtellina region (Northern Italy) during the 2009 vintage, were monitored by a Fourier Transform-near infrared (FT-NIR) and a -mid infrared (FT-MIR) spectrometer and by an electronic nose and tongue. The spectroscopic technique was used to investigate changes in the chemical composition of the must, while electronic nose and electronic tongue evaluated the evolution of the aroma and taste profile during the fermentation. Must-wine samples were also analyzed by traditional chemical methods in order to determine sugars (glucose and fructose) consumption and alcohol (ethanol and glycerol) production. PCA was applied to spectral, electronic nose and electronic tongue data, as an exploratory tool, to uncover modifications during the fermentation process. Furthermore, the chemical data and the first principal component scores from spectral, electronic nose and electronic tongue data were modeled as a function of time to identify critical points during fermentation. The results showed that through the modeling of NIR, MIR, electronic nose and electronic tongue data, these non destructive methods are suitable for the monitoring of grape must fermentation giving crucial information about the quality of the final product in agreement with chemical parameters. Although in this study the measurements were carried out in off-line mode (with its known drawbacks), in the future these non destructive techniques could be valid and simple tools, able to provide in-time information about the fermentation process and to assure the quality of wine.

An electronic nose coupled to gas chromatography was tested [24] to monitor alcoholic fermentation by Saccharomyces cerevisiae ICV-K1 and Saccharomyces cerevisiae T306, two strains well-known for their use in oenology. The biomass and ethanol concentrations and conductance changes were measured during yeast growth and allowed to observe the standard growth phases for both yeast strains. The two strains were characterized by a very similar tendency in biomass or ethanol production during the fermentation. E-nose was able to establish a kinetic of the production of aroma compounds production and which was then easy to associate with the fermentation phases. PCA showed that the data collected by e-nose during the fermentation mainly contained cultivation course information. Discriminant factor analysis (DFA) was able to clearly identify differences between the two strains using the four main principal components of PCA as input data. Nevertheless, the electronic nose responses being mainly influenced by cultivation course, a specific data treatment 
limiting the time influence on data was carried out and permitted to achieve an overall performance of $83.5 \%$.

NIR spectrometry and electronic nose data were used for on-line monitoring of yogurt and filmjölk (a Swedish yogurt-like sour milk) fermentations under industrial conditions [25]. The NIR and e-nose signals were selected by evaluation of PCA loading vectors and further analyzed by studying the variability of the selected principal components. First principal components for the NIR and the e-nose signals were used for on-line generation of a process trajectory plot visualizing the actual state of fermentation. The NIR signals were also used to set up empirical PLS models for prediction of the cultures' $\mathrm{pH}$ and titratable acidity (expressed as Thorner degrees, ${ }^{\circ} \mathrm{T}$ ). By using five or six PLS factors the models yielded acceptable predictions that could be further improved by increasing the number of reliable and precise calibration data. The presented results demonstrate that the fusion of the NIR and e-nose signals has a potential for rapid on-line monitoring and assessment of process quality of yogurt fermentation.

Measurement data from an electronic nose, a NIR spectrometer and standard bioreactor probes were utilized to follow the course of lab-scale yoghurt fermentation [26]. The sensor signals were fused using a cascade neural network: a primary network predicted quantitative process variables, including lactose, galactose and lactate; a secondary network predicted a qualitative process state variable describing critical process phases, such as the onset of coagulation or the harvest time. Although the accuracy of the neural network prediction was acceptable and comparable with the offline reference assay, its stability and performance were significantly improved by correction of faulty data. The results demonstrate that on-line sensor fusion with the chosen analyzers improves monitoring and quality control of yoghurt fermentation with implications to other fermentation processes.

In the study performed by Eklöv et al [27] a sensor array and pattern recognition routines (an electronic nose) were used to monitor a sausage fermentation in order to follow the changes in emitted volatile compounds during the fermentation process and to compare the electronic nose results with a sensory analysis. From the sensor array responses the fermentation time could be predicted using different methods, where principal component regression and an artificial neural network based on all sensors in the electronic nose performed best. A sensory panel evaluated the final product and these results were compared with the electronic nose measurements in the early stage of the process and on the final sausages. PCA data showed that one of the sausage batches clearly deviated from the other using both the sensory panel data and the 
electronic nose responses. The deviating batch was different already after $4 \mathrm{~h}$ and the difference was consistent during the process.

Finally, an on-line approach of non-invasive monitoring of the physiological changes in fermentation processes was presented by Bachinger and Mandenius [28]. In yeast batch and bacterial fed-batch fermentations it is shown that metabolic state changes can be revealed using an electronic nose. The transient responses of the gas sensors to the changes in the composition of the volatiles emitted from the cell cultures during fermentation are used to retrieve a semi-quantitative representation of the physiological state of the cultures. With the sensor responses of the electronic nose it is shown that physiological variables such as rates of growth, substrate uptake and product formation can be depicted. The non-invasive method thus seems as a pertinent alternative to conventional bioreactor monitoring methods.

\subsection{Using e-tongues}

Electronic tongues have been successfully applied to the monitoring of several components occurring during fermentation process such as those caused by specific microorganisms, production of food and beverages, as well as production of biogas. The main features of such applications are summarized in Table 2. Moreover, some of them are included in the interesting review devoted to the use of sensor systems for the monitoring of biotechnological process [12].

Regarding the sensor array used in the design of e-tongues, a wide variety of chemical sensors have been employed: electrochemical (potentiometric, voltammetric, amperometric, impedimetric and conductimetric), optical, mass, and enzymatic sensors (biosensors) [3]. As can be observed in Table 2, most of the systems utilized in fermentation monitoring use arrays of potentiometric sensors, especially ion-selective electrodes based on PVC membranes or chalcogenide glass, as well as combinations of both type of sensors. The main features of e-tongue applications shown in Table 2 are described thereafter.

The first paper on the application of e-tongues to the monitoring of fermentation process seems to be the one by Imamura et al. [29]. These authors used eight different lipid membranes in the multichannel taste sensor for monitoring the changes in the taste of miso (traditional Japanese soybean paste) during the fermentation process. The authors found that the responses of two of the sensors used increased linearly with the number of days of fermentation, whereas they had only a slight increase for ripe miso samples. Moreover, satisfactory correlation coefficients (higher than 0.87) between chemical parameters related to the fermentation length (amino-acid contents and titratable acidity) and the sensors output were obtained. 
Since the above commented study, some other works on the application of etongues to the monitoring of the production of other foodstuffs [30,31] as well as alcoholic beverages production processes such as winemaking [23] and brewing process [32] have been reported.

In the work by Kim et al. [30] the potential of a sensor array consisting of eight cation- and anion-selective polymer membrane electrodes to the monitoring of Kimchi, a Korean traditional pickle fermented with lactic acid bacteria, was studied. Samples were maturated for ten days at three temperatures $\left(4,10\right.$ and $\left.25^{\circ} \mathrm{C}\right)$ and analyzed with the sensor array. Additionally, titratable acidity of samples, a well established index for Kimchi fermentation, was determined. The authors observed that the response potentials of the sensor array increased during the fermentation period and storage temperatures. Moreover, for each storage temperature, PCA was performed using membrane potential values obtained from sensors. First principal component scoresfermentation time scatterplots showed similar profiles to those obtained for titratable acidiy-fermentation time scatterplots, mainly when using data obtained from the sensor group of cation-selective membranes and samples maturated at $4{ }^{\circ} \mathrm{C}$. These results suggest that the proposed sensor array could be useful for the monitoring of the titratable acidity changes occurring during Kimchi fermentations.

An e-tongue based on 30 non-specific potentiometric sensors was applied to the monitoring of a batch fermentation process of starting culture for cheese production [31]. PLS control charts allowed detection of fermentations running under 'normal' and 'abnormal' operating conditions at an early stage. Moreover, the capability of the etongue to quantify organic acids (such as citric, lactic, and orotic) in the fermentation media was demonstrated with average prediction errors in the $5-13 \%$ range.

Wine is a complex mixture of a great number of compounds arising from grape and fermentation, giving wine its interesting organoleptic properties. Compounds found in wine depend on the grape's genomic properties, but also by the strain of yeast used in fermentation, viticultural practices, the environment where the grapes are grown and the ageing in oak wood barrels [33]. Nowadays, a trained sensory panel is the only possible way to assess the flavor of wine. Flavor assessment by humans is inevitably associated with a number of serious drawbacks: (i) the subjectivity of the assessment; (ii) the score for particular sample depends greatly on the physical condition, health and mood of the panelist, even if that person is highly trained which often leads to irreproducible results. (iii) The fast blocking of human tongue taste receptors during a sensory session results in a very limited number of sensory assessments per day. (iv) Training and preparation of sensory experts are expensive and time-consuming [34]. In this context, e-tongues seem to be promising tools for an effective control at all stages 
of the winemaking and ageing process.

In the paper by Buratti et al. included in section 3.1 [23], for the monitoring of eight wine-must fermentation trials a commercial e-tongue (Taste-Sensing System SA 402B, Intelligent Sensor Technology Co., Ltd., Japan) was also used besides Fourier transform near and mid infrared spectroscopies and a e-nose. As commented previously, first principal component scores from spectral and electrochemical sensor data were modeled as a function of time to follow the kinetics of the fermentation process and to estimate crucial kinetic parameters. These estimates from spectral and e-nose data showed a good agreement with those obtained by the modeling of sugar consumption and alcohol production (correlation coefficients higher than 0.91). Though satisfactory, e-tongue data provided the lowest correlation coefficients (0.79-0.92).

Nowadays significant efforts are being directed to the development of instrumental methods for routine analysis of taste attributes of beer. Brewing and aging of beer are complex processes during which several parameters have to be controlled to ensure a reproducible quality of the finished product. These include chemical parameters that are measured with conventional analytical techniques and taste and aroma properties that are evaluated by the sensory panels [35].

Up till now research on the prediction of the sensory characteristics of beer from analytical techniques remains scarce, probably due to the complexity of the matrix and the variety of the components present therein. Beer constituents comprise more than 800 compounds and many of them contribute to its flavor characteristics. In the case of such a complex process of beer fermentation, it is difficult to choose the right techniques for classical instrumental analysis. Moreover, similarly to the other fermentation process, the application of analytical techniques can be problematic to estimate the final quality of the product, due to the rapid changes occurring throughout the beer fermentation process.

The potential of e-tongues as fast, simple and reliable analytical techniques to partly or fully replace the complex and expensive reference methods in the brewery industry is investigated in the work by Kutyła-Olesiuk et al. [32]. In this paper the application of a miniaturized hybrid e-tongue, combining both potentiometric and voltammetric sensors, to the monitoring of beer fermentation is presented. The analysis included samples from batch of homemade beer fermentation and from two stages of the process (fermentation reaction and maturation of beer). The obtained data were processed using partial least squares-discriminant analysis (PLS-DA). It was shown that the combination of potentiometric and voltammetric data provided lower classification error of samples according to their fermentation time or age and better quantitative prediction when compared to the use of data obtained from each 
electrochemical technique separately.

Regarding fermentations caused by specific microorganisms, Turner et al. [36] reported one the first studies related to the use of e-tongues in fermentation process monitoring. In this paper, an e-tongue consisting of 21 potentiometric chemical sensors was used for off-line measurements of model batch fermentation of Escherichia coli. Some physicochemical parameters were also determined at different fermentation times during the process using conventional analytical techniques and independently correlated with e-tongue output by means of PLS regression. It was possible to predict optical density, dry weight, acetate concentration and fermentation time with average prediction errors or $13,16,11$ and $5 \%$, respectively.

Legin et al. [37] proposed a potentiometric e-tongue system for the determination of ammonium, oxalate and citrate content in simulated fermentation media closely resembling real-world samples typical of a process involving Aspergillus niger. Data processing using artificial neural network (ANN) provided average relative prediction errors lower than $8 \%$. ANN provided slightly lower prediction errors than PLS regression for ammonium and citrate concentrations, most likely due to the non-linear dependence of sensor potentials on concentration. The device also allowed for quantifying the content of analytes in samples containing sodium azide, a compound commonly used to suppress microbial activity after sampling.

In the last few years, e-tongues have also been utilized for the monitoring of production of biogas [38-40]. In this biotechnological process, specific microorganisms under anaerobic conditions transform organic substrates present in waste into methane, which is used as a valuable energy source.

The research group of Ciosek et al. developed a miniaturized flow-through sensor array for the monitoring of methane fermentation of whey samples [38, 39]. PLS-DA inspection of data allowed for a satisfactory differentiation of samples gathered after 24 and 48 hours, but a partial overlapping of the clusters was observed and confirmed by the determination of chemical oxygen demand and volatile fatty acid content. However, precision was improved by splitting the dataset into two subsets according to their $\mathrm{pH}$ level [39]. In a further work [40], a novel design of ion selective electrodes was developed, which is fully compatible with the flow-through modules. In this case it was no necessary to split dataset to obtain satisfactory results.

Finally, it should also be remarked that the combination of an e-nose, an etongue, and an electronic eye is emerging as a promising tool for the on-line monitoring of food fermentation processes. It has already been applied to the characterization of red wines [41] and olive oils with different degree of bitterness [42]. The fusion of these 
three sensory modalities (volatiles, liquids, and color) has given rise to the so-called "electronic panel", which includes the corresponding sensors as well as the electronics and software necessary for combining information from the three modules. The simultaneous utilization of these devices increases the amount of information extracted, since visual aspects can also play an important role in the monitoring of the different compounds produced along a fermentation process. Therefore, there is no doubt that this innovative system will be of great utility in the field of food analysis, and more especifically in fermentations. 


\section{Conclusions and future trends}

In this paper, applications of sensor array systems -electronic noses and electronic tongues- to the monitoring of fermentation processes are reviewed. These devices are of particular interest for the analysis of complex gas and liquid mixtures due to some important advantages over conventional analytical techniques such as fast measurements, possibility of easy automation, relatively simple and inexpensive instrumentation, and the fact of being non-destructive techniques thus allowing an effective and continuous control at all stages of a fermentation process.

Electronic noses and tongues permit the possibility of classification of samples, as well as the quantitative (or at least semi-quantitative) determination of sample components simultaneously. They have also demonstrated a good potential for the assessment of the state and operation conditions of a fermentation process.

Although all the results presented in this review seem to be very promising, in our opinion these devices are still in an early stage of development, especially etongues, and much more research has to be carried out in order to implement them in the process production. Major concerns include the lack of intermediate precision studies (i.e. using different operators or instruments) and long term studies, more validation studies and higher number of analyzed samples being required in some cases to extract more reliable conclusions. Other practical issues such as sensor surface contamination, drift of responses and calibration stability have also to be addressed.

On the other hand, some differences between e-noses and e-tongues performance should be remarked. An advantage of e-noses is that the sampling systems used, mainly headspace techniques, avoid the direct contact between sensors and samples, thus reducing contamination of sensors. Nevertheless, some time is required to reach equilibrium between liquid and gas phases and, on the other hand, specially cleaning of a possible memory effect can also be an important drawback. Moreover, e-noses usually provide only semi-quantitative information, while e-tongues are straightforward with good capabilities for quantitative analysis.

In spite of these concerns, the future of e-nose and e-tongue seems to be encouraging, and upcoming trends include: the development of new chemosensitive materials and techniques for the preparation of sensors, the use of hybrid systems joining in the array of sensors of different nature, the inclusion of sensor arrays in flowbased analytical systems for real-time analysis, the fusion of e-nose and e-tongue techniques to improve the recognition capabilities of the system, and the coupling of 
sensor arrays with distributed expert systems for the advanced in-line monitoring of fermentation processes. 


\section{References}

[1] T.C. Pearce, S.S. Schiffman, H.T. Nagle, J.W. Gardner (Editors), in: Handbook of Machine Olfaction: Electronic Nose Technology, Wiley-VCH Verlag GmbH \& Co. KGaA, Weinheim, 2003.

[2] M. Peris, L. Escuder-Gilabert, Anal. Chim. Acta 638 (2009) 1-15.

[3] M. Biniecka, S. Caroli, TrAC Trend. Anal. Chem 30 (2011) 1756-1770.

[4] S. Sankaran, L.R. Khot, S. Panigrahi, Sens. Actuators B 171-172 (2012) 1-17.

[5] L. Escuder-Gilabert, M. Peris, Anal. Chim. Acta 665 (2010) 15-25.

[6] Y. Vlasov, A. Legin, A. Rudnitskaya, C. Di Natale, A. D’Amico, Pure Appl. Chem. 77 (2005) 1965-1983.

[7] A. Legin, A. Rudnitskaya, Y. Vlasov, Sensors Update, 10 (2002) 143-188.

[8] P. Ciosek, W. Wróblewski, Analyst 132 (2007) 963-978.

[9] A. Riul Jr., C.A.R. Dantas, C.M. Miyazaki, O.N. Oliveira Jr., Analyst 135 (2010) 2481-2495.

[10] M. del Valle, Electroanal. 22 (2010) 1539-1555.

[11] M. del Valle, Int. J. Electrochem. 2012 (2012) 1-11.

[12] A. Rudnitskaya, A. Legin, J. Ind. Microbiol. Biotechnol. 35 (2008) 443-451.

[13] A.D. Wilson, Sensors 13 (2013) 2295-2348.

[14] M. Ghasemi-Varnamkhasti, S.S. Mohtasebi, M.L. Rodríguez-Méndez, J. Lozano, S.H. Razavi, H. Ahmadi, Trends Food Sci. Technol. 22 (2011) 165-174.

[15] C. Pinheiro, C.M. Rodrigues, T. Schäfer, J.G. Crespo, Biotechnol. Bioeng. 77 (2002) 632-640.

[16] L. Marilley, S. Ampuero, T. Zesiger, M.G. Casey, Int. Dairy J. 14 (2004) 849856.

[17] N. Gutiérrez-Méndez, B. Vallejo-Cordoba, A.F. González-Córdova, G.V. Nevárez-Moorillón, B. Rivera-Chavira, J. Dairy Sci. 91 (2008) 49-57.

[18] W. Cynkar, D. Cozzolino, B. Dambergs, L. Janik, M. Gishen, Sens. Actuators B 124 (2007) 167-171.

[19] A.Z. Berna, S. Trowell, W. Cynkar, D. Cozzolino, J. Agric. Food Chem. 56 (2008) 3238-3244.

[20] N. Bhattacharya, S. Seth, B. Tudu, P. Tamuly, A. Jana, D. Ghosh, R. Bandhopadhyaya, M. Bhuyan, J. Food Eng. 80 (2007) 1146-1156.

[21] N. Bhattacharya, B. Tudu, A. Jana, D. Ghosh, R. Bandhopadhyaya, M. Bhuyan, Sens. Actuators B 131 (2008) 110-116.

[22] M. Sharma, D. Ghosh, N. Bhattacharya, Int. J. Eng. Sci. Invention 2 (2013) 5660. 
[23] S. Buratti, D. Ballabio, G. Giovanelli, C.M. Zuluanga Domínguez, A. Moles, S. Benedetti, N. Sinelli, Anal. Chim. Acta 697 (2011) 67-74.

[24] M. Calderón-Santoyo, P. Chalier, D. Chevalier-Lucia, C. Ghommidh, J.A. Ragazzo-Sanchez, Electron. J. Biotechn. 13 (2010) 1-12.

[25] M. Navratil, C. Cimander, C.F. Mandenius, J. Agric. Food Chem. 52 (2004) 415420.

[26] C. Cimander, M. Carlsson, C.F. Mandenius, J. Biotechnol. 99 (2002) 237-248.

[27] T. Eklöv, G. Johansson, F. Winquist, I. Lundström, J. Sci. Food Agric. 76 (1998) 525-532.

[28] T. Bachinger, C.F. Mandenius, Eng. Life Sci. 1 (2001) 33-42.

[29] T. Imamura, K. Toko, S. Yanagisawa, T. Kume, Sens. Actuators B 37 (1996) 179-185.

[30] N. Kim, K-r Park, I-S Park, Y-J Cho, Y.M. Bae, Biosens. Bioelectron 20 (2005) 2283-2291.

[31] K. Esbensen, D. Kirsanov, A. Legin, A. Rudnitskaya, J. Mortensen, J. Pedersen, L. Vognsen, S. Makarychev, Y. Vlasov, Anal. Bioanal. Chem. 378 (2004) 391395.

[32] A. Kutyła-Olesiuk, M. Zaborowski, P. Prokaryn, P. Ciosek, Bioelectrochemistry 87 (2012) 104-113.

[33] A.P. Umali, S.E. LeBoeuf, R.W. Newberry, S. Kim, L. Tran, W.A. Rome, T. Tian, D. Taing, J. Hong, M. Kwan, H. Heymann, E.V. Anslyn, Chem. Sci. 2 (2011) 439-445.

[34] D. Kirsanov, O. Mednova, V. Vietoris, P.A. Kilmartin, A. Legin, Talanta 90 (2012) 109-116.

[35] A. Rudnitskaya, E. Polshin, D. Kirsanov, J. Lammertyn, B. Nicolai, D. Saison, F.R. Delvaux, F. Delvaux, A. Legin, Anal. Chim. Acta 646 (2009) 111-118.

[36] C. Turner, A. Rudnitskaya, A. Legin, J. Biotechnol. 103 (2003) 87-91.

[37] A. Legin, D. Kirsanov, A. Rudnitskaya, J.J.L. Iversen, B. Seleznev, Talanta 64 (2004) 766-772.

[38] P. Ciosek, A. Buczkowska, E. Witkowska, W. Wróblewski, IEEE Sensors 2009 Conference 1-3 (2009) 1502-1505.

[39] A. Buczkowska, E. Witkowska, Ł. Górskia, A. Zamojska, K.W. Szewczyk, W. Wróblewski, P. Ciosek, Talanta 81 (2010) 1387-1392.

[40] E. Witkowska, A. Buczkowska, A. Zamojska, K.W. Szewczyk, P. Ciosek, Bioelectrochemistry 80 (2010) 87-93.

[41] M.L. Rodríguez-Méndez, A.A. Arrieta, V. Parra, A. Bernal, A. Vegas, S. Villanueva, R. Gutiérrez-Osuna, IEEE Sensors Journal 4 (2004) 348-354. 
[42] C. Apetrei, I.M. Apetrei, S. Villanueva, J.A. de Saja, F. Gutiérrez-Rosales, Anal. Chim. Acta 663 (2010) 91-97. 
Abbreviations:

- 4EG: 4-ethylguaiacol

- 4EP: 4-ethylphenol

- ANN: artificial neural network

- CG: gas chromatography

- CP: conducting polymer

- CPE: carbon paste electrode

- DFA: discriminant factor analysis

- FT: Fourier transform

- HPLC: high performance liquid chromatography

- IMS: ion mobility spectrometry

- MIR: mid infrared spectroscopy

- MLR: multiple linear regression

- MOS: metal oxide semiconductor

- MOSFET: metal oxide semiconductor field-effect transistor

- MS: mass spectrometry

- NIR: near infrared spectroscopy

- PCA: principal component analysis

- PLS: partial least squares

- PLS-DA: partial least squares-discriminant analysis

- SHS: static headspace

- SLDA: stepwise linear discriminant analysis

- SOM: self-organizing map

- SPME: solid-phase microextraction

- TDNN: time-delay neural network

- VOCs: volatile organic compounds 
Figure Captions

Figure 1. Schematic representation of the components of an electronic nose (a) and an electronic tongue (b). 


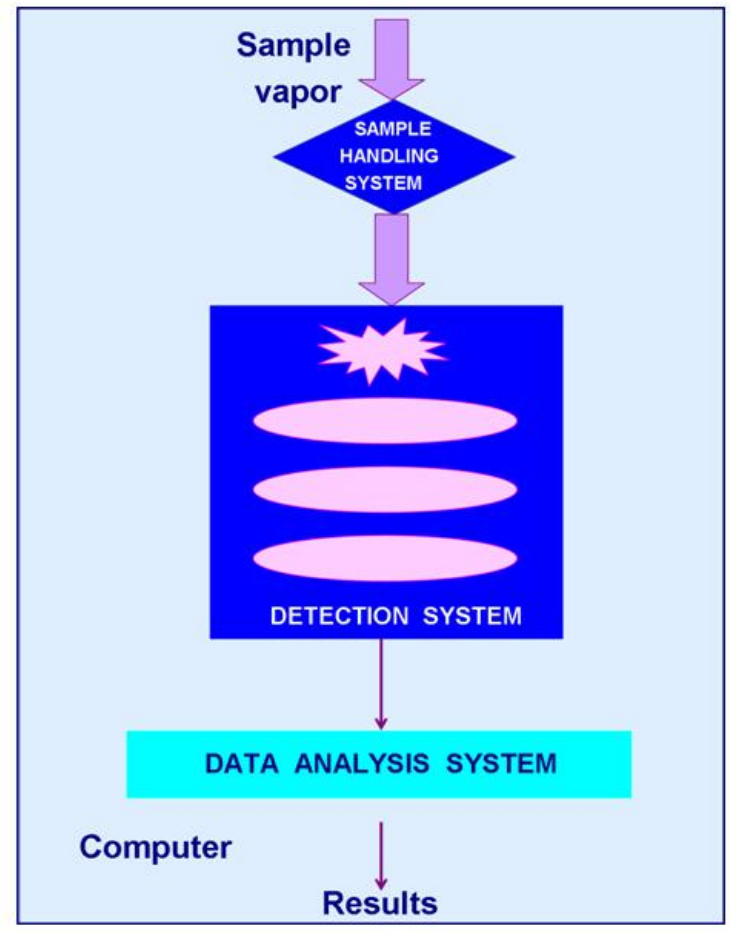

(a)

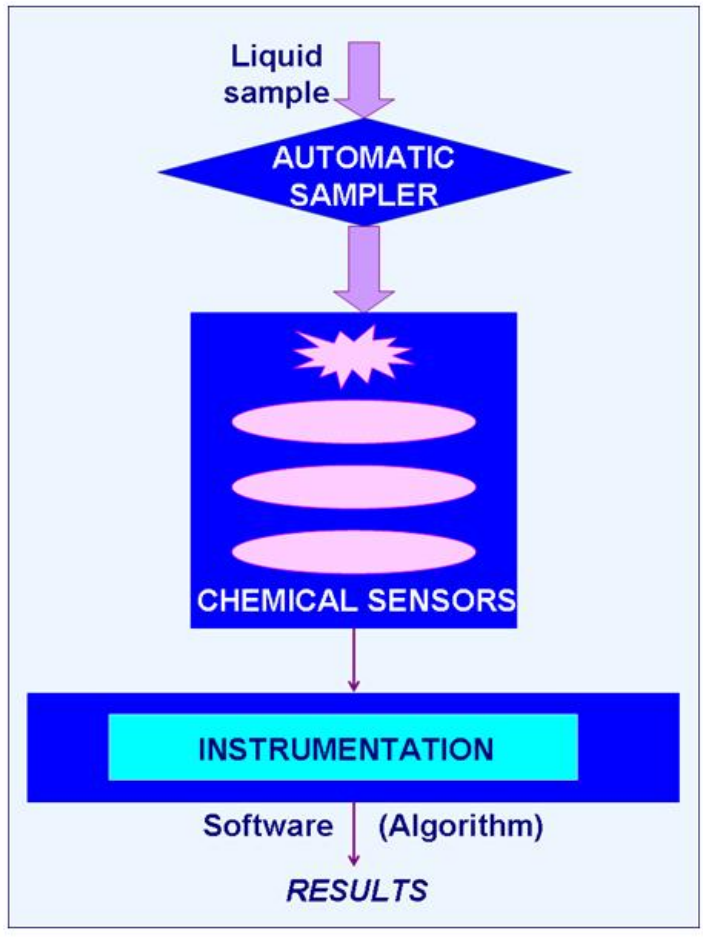

(b) 
Table 1

Main applications of e-noses in fermentation monitoring

\begin{tabular}{|c|c|c|c|c|c|}
\hline Sample & Type of study & Sample handling system & Sensors & $\begin{array}{l}\text { Data processing } \\
\text { algorithm }\end{array}$ & Ref. \\
\hline Wine-must & Discrimination between fermentation stages & SHS - pervaporation & $32 \mathrm{CPs}(\mathrm{A} 32 \mathrm{~S} \text { AromaScan })^{a}$ & PCA & {$[15]$} \\
\hline $\begin{array}{l}\text { Milk fermented with Lactobacillus casei } \\
\text { strains used in Gruyère cheese }\end{array}$ & Discrimination between genotype strains & INDEX & MS (Smart Nose $\left.{ }^{\circledR}\right)^{b}$ & PCA & {$[16]$} \\
\hline $\begin{array}{l}\text { Milk fermented with Lactococcus lactis } \\
\text { strains }\end{array}$ & Discrimination between odor intensity scores & SHS & $12 \operatorname{MOS}(\mathrm{FOX} 3000)^{\mathrm{C}}$ & PCA & {$[17]$} \\
\hline Australian red wines & Spoilage caused by Brettanomyces yeast & SHS & MS (HP4440) ${ }^{d}$ & $\begin{array}{l}\text { PCA, PLS, } \\
\text { SLDA }\end{array}$ & [18] \\
\hline Australian red wines & Spoilage caused by Brettanomyces yeast & $\begin{array}{l}\text { SPME (for MOS) } \\
\text { SHS (for MS) }\end{array}$ & $\begin{array}{l}\text { FOX } 3000 \\
\text { HP4440 }\end{array}$ & PLS & [19] \\
\hline Black tea & Estimation of optimum fermentation time & SHS & $8 \mathrm{MOS}$ & TDNN, SOM & [20-22] \\
\hline Italian red wine & Evolution of aroma profile & SHS & 10 MOS (PEN2) ${ }^{\mathrm{e}}$ & PCA & [23] \\
\hline $\begin{array}{l}\text { Synthetic media containing two } \\
\text { Saccharomyces cerevisiae strains }\end{array}$ & $\begin{array}{l}\text { Identification of two strains along an alcoholic } \\
\text { fermentation }\end{array}$ & SHS & $18 \operatorname{MOS}(F O X 4000)^{c}$ & PCA, DFA & {$[24]$} \\
\hline Yogurt and filmjölk (yogurt-like sour milk) & $\begin{array}{l}\text { Rapid on-line monitoring of yogurt } \\
\text { fermentation }\end{array}$ & SHS & $\begin{array}{l}10 \text { MOSFET (with catalytic gates of } \mathrm{Pd} \text {, } \\
\text { Ir and } \mathrm{Pt} \text { ), } 19 \mathrm{MOS} \text { and } 1 \text { infrared } \mathrm{CO}_{2} \\
\text { sensor }\end{array}$ & PCA, PLS & [25] \\
\hline Fat milk fermented & $\begin{array}{l}\text { Sensor fusion for on-line monitoring of yogurt } \\
\text { fermentation }\end{array}$ & SHS & $\begin{array}{l}10 \text { MOSFET (with catalytic gates of } \mathrm{Pd} \text {, } \\
\text { Ir and } \mathrm{Pt} \text { ) and } 19 \mathrm{MOS}\end{array}$ & ANN, PCA & [26] \\
\hline Homemade sausages & $\begin{array}{l}\text { Early prediction of final quality of fermented } \\
\text { sausage }\end{array}$ & SHS - injection & $\begin{array}{l}10 \text { MOSFET ((with catalytic gates of } \\
\mathrm{Pd}, \text { Ir and } \mathrm{Pt}) \text { and } 4 \mathrm{MOS}\end{array}$ & PCR, ANN, PCA & [27] \\
\hline $\begin{array}{l}\text { Synthetic media with Saccharomyces } \\
\text { cerevisiae and Escherichia coli }\end{array}$ & $\begin{array}{l}\text { Monitoring of physiological changes in } \\
\text { fermentation processes }\end{array}$ & SHS - gas flow injection & $\begin{array}{l}\text { In yeast batch fermentations: } 10 \\
\text { MOSFET (with catalytic gates of } \mathrm{Pd} \text {, Ir } \\
\text { and } \mathrm{Pt} \text { ) and } 4 \text { MOS } \\
\text { In E. coli fed-batch fermentations: } 10 \\
\text { MOSFET, } 19 \text { MOS and } 1 \text { infrared } \\
\text { sensor }\end{array}$ & ANN, PLS & {$[28]$} \\
\hline
\end{tabular}

For details see Abbreviations section

${ }^{\text {a }}$ A32S AromaScan is a conducting polymer (CP) based e-nose commercially available from Osmetech plc, UK

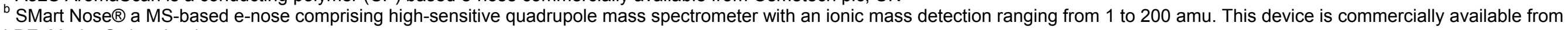
LDZ, Marin, Switzerland

${ }^{c}$ FOX 3000 and FOX 4000 are e-noses based on metal oxide semiconductors (MOS) sensors. They are commercially available from Alpha MOS, Toulouse, France

${ }^{d}$ HP 4440 is a MS-based e-nose commercially available from Agilent Technologies

${ }^{\mathrm{e}} \mathrm{PEN} 2$ is a portable electronic nose based on MOS sensors. It is commercially available from Win Muster Airsense Analytics Inc., Schwerin, Germany 
Table 2

Main applications of e-tongues in fermentation monitoring

\begin{tabular}{|c|c|c|c|c|}
\hline Sample & Type of study & Chemical sensors & $\begin{array}{l}\text { Data processing } \\
\text { algorithm }\end{array}$ & Ref. \\
\hline Miso (soybean paste) & $\begin{array}{l}\text { Modeling and prediction of amino-acids contents and titratable } \\
\text { acidity of samples with different fermentation degrees }\end{array}$ & $\begin{array}{l}\text { Potentiometric sensors (8 plasticized PVC sensors containing lipid } \\
\text { membranes; } \mathrm{Ag} / \mathrm{AgCl} \text { reference electrode) }\end{array}$ & MLR & [29] \\
\hline Kimchi & $\begin{array}{l}\text { Assessment of changes of the titratable acidity of samples during } \\
\text { fermentation }\end{array}$ & $\begin{array}{l}\text { Potentiometric sensors ( } 8 \text { plasticized PVC sensors containing } \\
\text { polymer membranes; Ag/AgCl reference electrode) }\end{array}$ & PCA & [30] \\
\hline $\begin{array}{l}\text { Starting culture for light } \\
\text { cheese production }\end{array}$ & $\begin{array}{l}\text { Discrimination of samples from fermentation batches } \\
\text { Modeling and prediction of organic acids and peptide profile }\end{array}$ & $\begin{array}{l}\text { Potentiometric sensors ( } 30 \text { chalcogenide glass and solvent } \\
\text { polymeric working electrodes; } \mathrm{Ag} / \mathrm{AgCl} \text { reference electrode) }\end{array}$ & PLS & [31] \\
\hline Must-wine & $\begin{array}{l}\text { Discrimination of samples according to their fermentation time } \\
\text { Modeling and prediction of kinetics of wine-must fermentation } \\
\text { process }\end{array}$ & Commercial e-tongue (4 sensors with artificial lipid membranes) & PCA & [23] \\
\hline Beer & $\begin{array}{l}\text { Monitoring of the fermentation and beer aging process, prediction } \\
\text { of fermentation time }\end{array}$ & $\begin{array}{l}10 \text { miniaturized ion-selective electrodes (potentiometric e-tongue) } \\
\text { and silicon based } 3 \text {-electrode voltammetric transducers } \\
\text { (voltammetric e-tongue) }\end{array}$ & $\begin{array}{l}\text { PLS-DA } \\
\text { MLR (backward } \\
\text { stepwise method) }\end{array}$ & [32] \\
\hline $\begin{array}{l}\text { Synthetic media with } \\
\text { Escherichia coli }\end{array}$ & $\begin{array}{l}\text { Modeling and prediction of physicochemical parameters during } \\
\text { the fermentation process }\end{array}$ & $\begin{array}{l}\text { Potentiometric sensors ( } 21 \text { chalcogenide glass and plasticized } \\
\text { PVC working electrodes; } \mathrm{Ag} / \mathrm{AgCl} \text { reference electrode) }\end{array}$ & PLS & [36] \\
\hline $\begin{array}{l}\text { Model solutions where } \\
\text { Aspergillus niger are } \\
\text { typically grown }\end{array}$ & $\begin{array}{l}\text { Modeling and prediction of organic acids and ammonium } \\
\text { concentrations during the fermentation process }\end{array}$ & $\begin{array}{l}\text { Potentiometric sensors ( } 8 \text { plasticized PVC sensors, chalcogenide } \\
\text { glass sensors, glass } \mathrm{pH} \text { electrode; } \mathrm{Ag} / \mathrm{AgCl} \text { reference electrode) }\end{array}$ & ANN, PLS & [37] \\
\hline Whey & $\begin{array}{l}\text { Methane fermentation monitoring } \\
\text { Discrimination between fermentation times } \\
\text { Prediction of chemical oxygen demand and volatile fatty acid } \\
\text { contents }\end{array}$ & $\begin{array}{l}\text { Potentiometic sensors ( } 10 \text { solid-state electrodes with various types } \\
\text { of PVC membranes doped with electroactive components; } \\
\mathrm{Ag} / \mathrm{AgCl} \text { reference electrode) }\end{array}$ & $\begin{array}{l}\text { PLS-DA } \\
\text { PLS }\end{array}$ & {$[38,39]$} \\
\hline Whey & $\begin{array}{l}\text { Methane fermentation monitoring } \\
\text { Prediction of chemical oxygen demand and volatile fatty acid } \\
\text { contents }\end{array}$ & $\begin{array}{l}\text { Potentiometic sensors ( } 10 \text { ion selective electrodes with various } \\
\text { types of PVC membranes doped with electroactive components; } \\
\text { Ag/AgCl reference electrode) }\end{array}$ & PLS & [40] \\
\hline
\end{tabular}

For details see Abbreviations section 